\title{
interview
}

\section{Controlling quantum flow}

\author{
Achieving dynamic optical control of exciton polariton quantum flow could provide a deeper \\ understanding of quantum behaviour and inspire new types of device, says Daniele Sanvitto from the \\ Istituto Nanoscienze in Italy.
}

\section{What is quantum flow?}

Quantum flow is a phenomenon in which the state of a fluid can be described by an order parameter. Classical flow can be considered to be the movement of single particles with different kinetic momenta. Quantum flow, in contrast, is described as an ensemble of particles that moves as a single entity. Quantum flow occurs because particles feel their quantum nature at very small interparticle distances. In our work, we investigated the quantum flow of exciton polaritons - quasiparticles made by coupling excitons with photons which were created by injecting light from a Ti:sapphire laser into a semiconductor microcavity containing InGaAs quantum wells. The advantage of studying quantum flows using exciton polaritons rather than superfluid helium and atomic Bose-Einstein condensates is the ease with which they can be generated, manipulated and detected.

\section{What is your motivation behind} this research?

Photons in a vacuum do not interact with each other. Exciton polaritons, on the other hand, interact with each other while also retaining the desirable properties of photons. This is an important feature that could be useful for realizing threshold-less lasers and quantum optical devices. The fundamental physics of quantum hydrodynamic effects and topological excitations such as vortices has received increasing attention in recent years. A couple of years ago, we demonstrated that exciton polaritons could behave as a superfluid. The superfluidity was broken when the fluid velocity exceeded the critical velocity, which raised questions about the boundary between superfluid and nonsuperfluid regimes. We then considered how quantum flow could be controlled. Previous studies have shown that quantum flows of exciton polaritons can move towards natural defects in semiconductor microcavities, and that laser light can be used to nucleate quantized vortices. However, in these schemes the researchers were unable to control the quantum flow dynamically, and that is what we set out to achieve. Our work is the first experimental demonstration of the optical dynamic control of quantum flow.

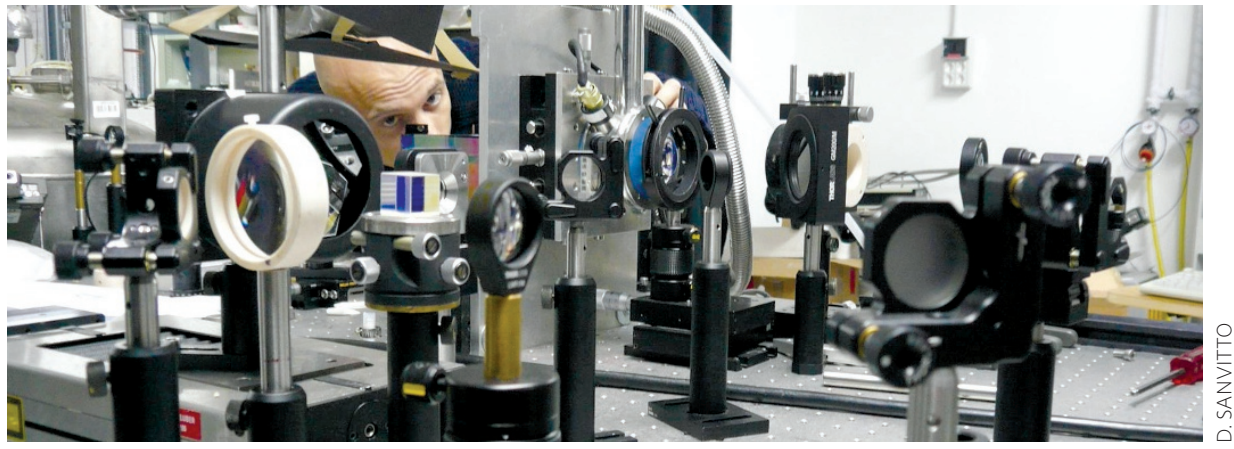

The use of light beams to manipulate and study quantum fluidic effects such as superfluidity could provide new insights to fundamental physics.

How did you get the idea of optically controlling quantum flow?

We found that strong laser excitation caused the potential landscape of exciton polaritons to change significantly, which suggested that a pulsed laser could be used to induce exciton polariton flow and a continuous-wave laser could be used to alter the local potential sensed by the flowing exciton polaritons. One problem associated with this was the phase of the pulsed laser, which can strongly affect the quantum flow of exciton polaritons. To overcome this issue, we masked half of the laser spot, which allowed us to create and move exciton polaritons using a pulsed laser and change the shape and depth of the potential landscape - seen by the flow with a continuous-wave laser. This is a very useful technique for studying and controlling the dynamics of quantum flow.

\section{What challenges did you need} to overcome?

High crystalline quality in the semiconductor microcavity is absolutely imperative. Realizing optical control requires the quantum wells, cavity and mirrors to be prepared with very low natural defects. The lasers needed to be of orthogonal polarization to avoid any interaction between the two exciton polariton populations. We also needed a picosecondtimescale observation system, as the flow speed was around $1 \%$ of the speed of light. We achieved this by interfering the emission with a reference beam, thus providing time-resolved real-space interferograms for measurement using a synchroscan streak camera. These experiments could not have been implemented without collaborative efforts from experimentalists at the University Pierre and Marie Curie and the Centre National de la Recherche Scientifique in France and theorists at the University of Paris and the Istituto Nazionale di Ottica of the Consiglio Nazionale delle Ricerche in Trento, Italy.

\section{Do you have plans for future work in this area?}

There are still many things to do. One of the most interesting ideas is to reduce the density of the polaritons. A quantized vortex comprising only one polariton would be extremely useful for studying quantum computation. Another goal is to create a polariton transistor based on a quantum fluid, which could allow the signal from exciton polaritons to be amplified using interactions with other polaritons. Devices working with polaritons would be energetically favourable, have low or no dissipation, and operate without the need for active elements to convert light into electric currents. However, realizing such devices is a significant challenge, and there are many hurdles to overcome before it can become a reality.

\section{INTERVIEW BY NORIAKI HORIUCHI}

Daniele Sanvitto and co-workers have a Letter on the optical control of quantum flow on page 610 of this issue. 